\title{
Antibiotics using in Georgia: clinical and epidemiological features
}

\author{
Korinteli I., Javakhadze M., Pagava K.
}

Tbilisi State Medical University, Tbilisi, Georgia

Background. High incidence and lethality of infectious disease is an actual problem of the 21st century medicine. Infectious diseases are one of the most common causes of morbidity worldwide. Untreatable bacterial infections with the current available antibiotics lead to increased morbidity and mortality. Antimicrobials are the most commonly prescribed drugs in the community and hospital setting. However, inappropriate use of antibiotics in recent years has particularly increased the number of antibiotic-resistant strains. Antimicrobial resistance is a significant global health threat. Without effective antimicrobials, much of the progress made in fighting infectious disease would be lost. The recent rise in resistance to these critical medicines is therefore extremely worrisome.

Objective. To study epidemiological and clinical aspects of antibiotics using in Georgia.

Materials and methods. The study was retrospectiveprospective. We used ATC/DDD methodology for epidemiological research of antibiotic using in the country and it held 2011-2019. According to this methodology, we calculate defined daily doses of antibiotics per 1000 inhibitors (DID). The point prevalence survey was performed in 20172019 in Georgian hospitals for clinical research.

Results and discussion. In last year increased antibiotic using in Georgian population. The lowest rate was in 2014 (19 DID) and the highest in 2019 (37 DID). The most commonly used antibiotics in Georgia are ceftriaxone, amoxicillin / clavulanic acid, azithromycin and ciprofloxacin. Frequency of antibiotic treatment in Georgian hospitals ranges between $77.6 \%$ (2017) and $86.1 \%$ (2019). The use of third generation cephalosporin ranges between $61.9 \%$ (2017) and $59.6 \%$ (2019). From the clinical aspects of antibiotic therapy, it is noteworthy that prevalence of antibiotic using in Georgian clinics is high and ranged from $77.6 \%$ (2017) to $86.1 \%$ (2019). Antibacterial prophylactic decreased and ranged from $38.3 \%$ (2017) to $20.3 \%$ (2019). Antibiotic selection according to guidelines on the most frequent nosology ranges between $73.8 \%$ (2017) and $69.2 \%$ (2019). Empirical antibiotic therapy decreased in last years and ranges between $92.2 \%$ (2017) and $69.2 \%$ (2019).

Conclusions. Increasing rate of antibiotic using in Georgian population and high use of broad-spectrum antibiotics in remarkable. In hospitals, antibiotics are prescribing according to the national or intra hospital guidelines, but there prevalence rate is high. Patient histories included information about general criteria of starting antibiotic therapy. Stop or review date of antibiotic treatment after $72 \mathrm{~h}$ in poorly presented. We think that it is very important to continue epidemiological and clinical research for future. Also update or developed new guidelines will be perfect way for optimization.

Key words: infectious diseases, epidemiology of antibiotics. 